\title{
A Study on the Effects of Facial Expression Enhancement Using a Movable Ear-Type Device Linked to Eyebrows Using Facial Expression Analysis
}

\author{
Shoko KIMURA*, Ayaka FUJII ** and Kazunori MIYATA * \\ * Japan Advanced Institute of Science and Technology, 1-1 Asahidai, Nomi-shi, Ishikawa 923-1292, Japan \\ shokokm@ieee.org,miyata@jaist.ac.jp \\ ** The University of Tokyo, 7-3-1 Hongo, Bunkyo-kui, Tokyo 113-8654, Japan \\ a-fujii@jsk.imi.i.u-tokyo.ac.jp
}

\begin{abstract}
Nonverbal information can be used to convey emotions. In some cases, we can read emotions from changes in the other person's facial expressions. However, the facial expression is not transmitted to other people in some cases. To address this issue, a glasses-type device with pseudo eyebrows that change eyebrow's thickness and angle was used in the previous research to facilitate the transmission of emotions to others. The device is charging type and cannot be used easily and cannot work for one day. In this study, we use "SUGO-MIMI," a lightweight device that does not require a power source to extend facial expressions by eyebrow movements. The SUGO-MIMI extends the eyebrow movements by connecting the eyebrows to thin plates imitating cat ears attached to the headband with wires and linking the eyebrows' movement to the plates. The eyebrow movement is extended. Thus, the emotions of joy, anger, and sorrow can be emphasized. As a result of analyzing the facial expressions of the subjects that watch videos of a person who wears the SUGO-MIMI using the Microsoft Face API, we obtained that the SUGO-MIMI can amplify some emotions and that women tended to have more positive impressions of the SUGO-MIMI than men.
\end{abstract}

Keywords: Emotion recognition, Facial expression expansion, Human augmentation, Cognitive psychology, Communication

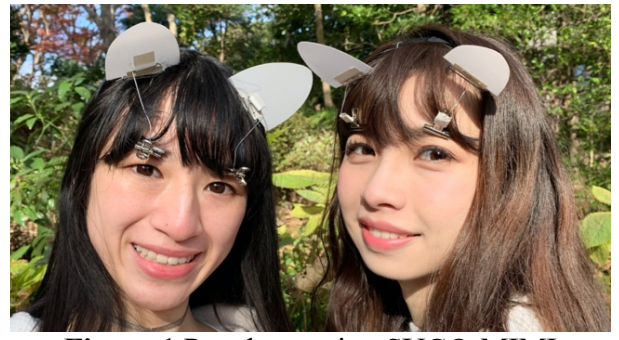

Figure 1 People wearing SUGO-MIMI

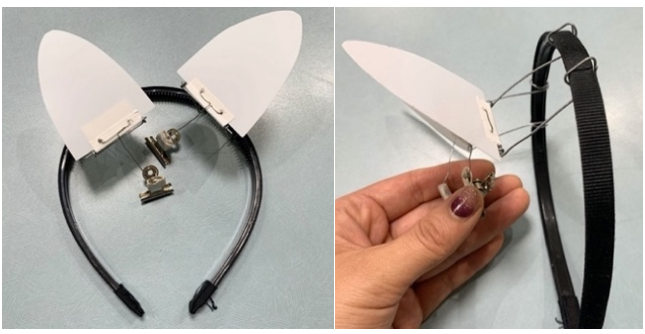

Figure 2 Overview of SUGO-MIMI

\section{INTRODUCTION}

Nonverbal communication through facial expressions and gestures are as important as verbal communication in emotional transmission [1]. However, some people are not good at reading facial expressions due to their developmental disabilities and lack of facial expression. According to Hidaka et al., it is necessary to accept the facial expressions' signals to read between the lines [2]. Animals such as dogs and cats, cannot use language to communicate; they express their emotions through cries, ears, and tails [3]. Besides, Juliane et al. discovered that dogs' facial expressions, including their eyebrows, indicate the degree of attachment between humans and dogs when making eye contact with humans [4]. What can be said from these examples is that expressing emotions through facial expressions can be used to communicate one's state to others in an easy-to-understand manner. It can also be used to make decisions for the recipient of the expression. Facial expressions are closely related to the criteria for judgment of people and can be a way to convey a state that cannot be fully conveyed by language. With expressing emotions through facial expressions, Matsui et al. assumed that emotions are more easily conveyed when the eyebrows are deformed and proposed an eyeglass-type device that displays eyebrows [5]. In this device, the eyebrows' thickness and the vertical position of the eyebrow ends were changed using thermochromic ink. It is difficult to wear this device continuously since it is driven by a battery. From the device's perspective for visualizing human's emotions, neurowear ${ }^{l}$ developed necomimi for expressing emotions clearly with ears like animals [6]. This device calculates emotional information from brain waves acquired by a sensor attached to the forehead. It also reflects the emotional information of joy, anger, sorrow, and pleasure in the movements of the ears

\footnotetext{
${ }^{1}$ neurowear is a Japanese product team that creates products using emotions. .
} 
of a cat. KANSEI analyzer [7] used graphs and marks to display the emotions obtained from brain waves. However, these devices have the problem that there is some delay in processing the brain waves, and the production cost is high.

In this study, we propose a lightweight and easy-tohandle device that does not require a power source. We assume that the movement, which is linked to the eyebrows has effects similar to those in the previous research [5]. We investigate the device effect, a hairband, which is equipped with a rounded triangular plate (hereinafter called the movable ear part) that moves up and down with the eyebrow movements. We call this hairband "SUGO-MIMI." The movable ear part has one side composed of a short straight line and two sides that are long and round lines, like an isosceles triangle. To avoid giving an unpleasant impression, this shape like pet ears was chosen. Figure 1 shows a photo of people wearing SUGO-MIMI. An overview of the structure is shown in Figure 2. We expect that SUGO-MIMI enhances and expands human facial expressions to make it easier for people to read emotions and communicate smoothly. We also evaluate the impressions of SUGO-MIMI and verify the effectiveness of the proposed method.

\section{EXPERIMENTAL DESIGN}

Though the movement of the mouth corners is the easiest way to convey emotions, people around the world have been covering their mouths with masks due to the COVID-19 in 2020, and it has become difficult to see the movement of the mouth corners. The other parts of the face that can be used to judge emotions are the eyes and eyebrows. We can judge emotion by the movement of the eyes and the frequency of blinking. For items that can be attached directly to the eyes, no one's other than false eyelashes is common. False eyelashes are difficult for many men, small children, and the elderly to attach since they have never used them before. Therefore, we used SUGO-MIMI, which focuses on extending the up and down movement of the eyebrows and is simple and easy to attach.

\subsection{Device Proposals and Systems}

In SUGO-MIMI, a hairband and movable ear parts made of lightweight plastic boards are fixed with wires. The movable ear parts tilt on the axis of this fixed part. To move the ear part, a hole is made in the short side of the movable ear part and attach another thin wire to it. When this wire moves, the short side of the movable ear part is moved, and the plate rotates around a fixed axis. A metal clip is attached to the other end of the wire to move the short side of the movable ear part through a wire. This clip is used for clipping the eyebrows. After clipping the eyebrows and adjusting the positional relationships of the hairband, head and eyebrows, the movable ear parts stand up straight above the head. By this mechanism, the ear parts move back and forth as the eyebrows move up and down (Figure3).

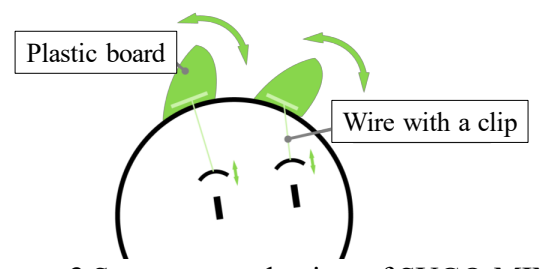

Figure 3 Structure mechanism of SUGO-MIMI

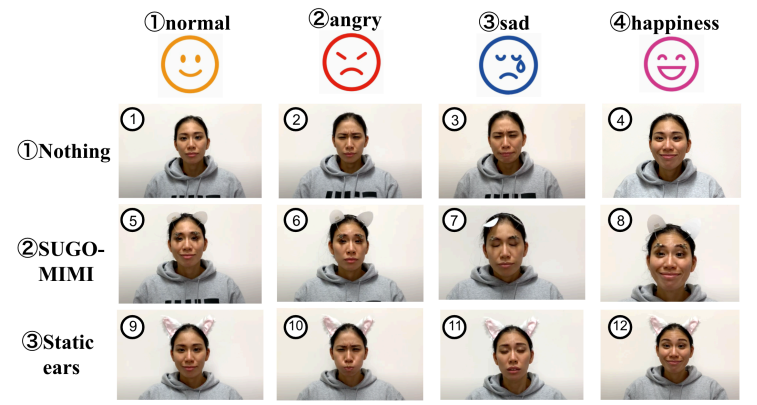

Figure4 Facial expressions in videos of the experiment

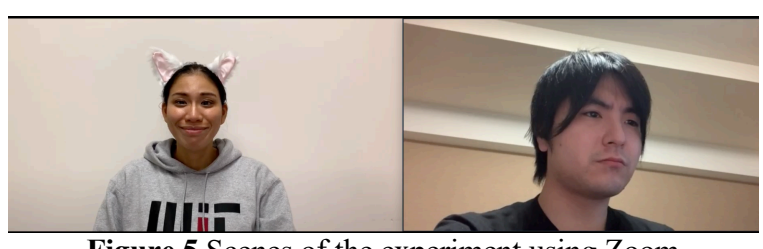

Figure 5 Scenes of the experiment using Zoom

\section{IMPRESSION EVALUATION}

To evaluate the effect of SUGO-MIMI, we compare the impressions with and without it. Among the six universal facial expressions proposed by Ekman et al. [8]: anger, disgust, fear, happiness, sadness, and surprise, we evaluate happiness, sadness, and anger. Since happiness can be categorized as a favorable facial expression and disgust, fear and sadness can be categorized as negative, we analyze these three facial expressions: happiness (favorable), sadness (negative), and anger.

We recorded 12 30-s videos of the four facial expressions (normal, angry, sad, and happy) in three states (with SUGO-MIMI in motion, with rounded triangular ears that do not move, and with nothing attached). A part of the videos is shown in Figure 4.

The experiment was conducted on Zoom that is the remote conference system, and the participants are presented with the 12 types of videos in numbered order described in Figure 4. They answer the questionnaires immediately after watching the videos of each condition (every 30 seconds).

The questionnaires took approximately 60 seconds or less to be completed, and the entire experiment was conducted for 20 minutes. We also recorded the status of the online participants using Zoom. The total number of participants was 67 . Figure 5 shows the experimental scene. The left side of the figure shows the video used in the experiment, and the right side shows a participant watching the video.

\subsection{Facial Expression Analysis Using Face API}

We conducted an emotional analysis comparison of the facial expressions of the subjects who watched the videos used in this experiment. We used three face images 
extracted from around 5, 15, and 25 seconds out of the 30 seconds that the subject was watching the video and extracted a total of 36 images from 12 videos. The images were sampled with regularity based on the method by Shimoda et al. [9]. After excluding the recording errors and some subjects who could not be analyzed because their faces were partially out of the camera's field of view, the total number of participants subjected to facial expression analysis was 52 (30 women and 22 men). The total number of data was 1872 . The data were analyzed for eight types of emotions (anger, contempt, disgust, fear, happiness, neutral, sadness, and surprise) by Microsoft Face API, referring to the previous research [10].

\section{EXPERIMENTAL RESULTS}

Figures 6, 7, and 8 show the percentages of the eight types of emotions. We analyzed the subjects in three patterns: whole, males, and females subjects and compared their results. The vertical numbers $1-12$ in the graphs correspond to the numbers of the 12 videos shown in Figure . No ears condition, which is the condition of the video of a person with nothing attached corresponds to 14. SUGO-MIMI condition, which is the condition of the video of a person with SUGO-MIMI which moves up and down according to the emotion corresponds to 5-8. Static ears condition, which is the condition of the video of a person with static animal ears which do not move by emotions corresponds to 9-12.

\subsection{Whole subjects}

Figure 6 shows the comparison results of the whole subjects' emotions. Based on happiness, which had the highest ratio other than neutral, the largest change was in 8 (happy expression in the SUGO-MIMI condition) with $12.2 \%$, followed by 4 (happy expression in the no ears condition) with $11.1 \%$. For all the four facial expressions, the percentage of happiness was higher in the SUGOMIMI condition than in the static ears or no ears conditions, suggesting that the presence of SUGO-MIMI amplified and conveyed the joy.

The second highest percentage was in sadness. The most significant change was 11 (sad expression in the static ears condition) with $5.98 \%$, followed by 7 (sad expression in the SUGO-MIMI condition) with 5.77\%. In the sad expression, the sadness was amplified more in the condition with some ears on the head than in the no ears condition.

The third most significant change was in contempt. The highest ratio was 6 (angry expression in the SUGO-MIMI condition) with $1.09 \%$, followed by 11 (sad expression in the static ears condition) with $0.849 \%$. In the case of sad and angry expression, the values tended to be larger in the condition with ears on the head than in the no ears condition.

\subsection{Male Subjects}

Figure 7 shows emotion comparison results in male subjects. Based on sadness, which had the highest ratio other than neutral, the largest change was 5 (normal

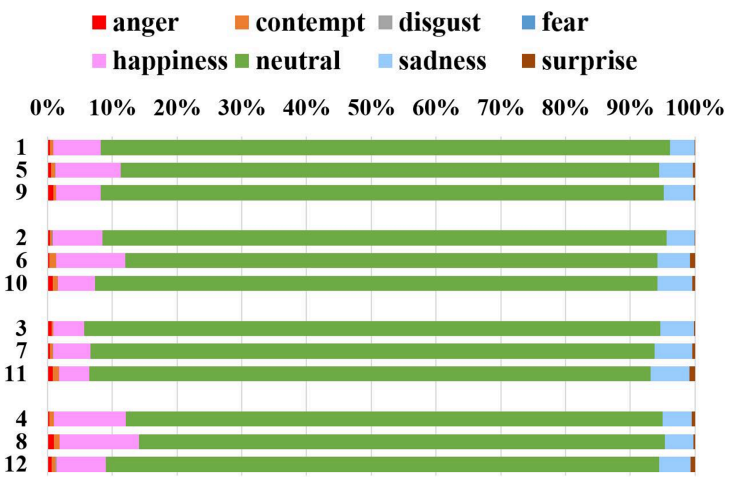

Figure 6 Whole subjects' 8 emotions comparison

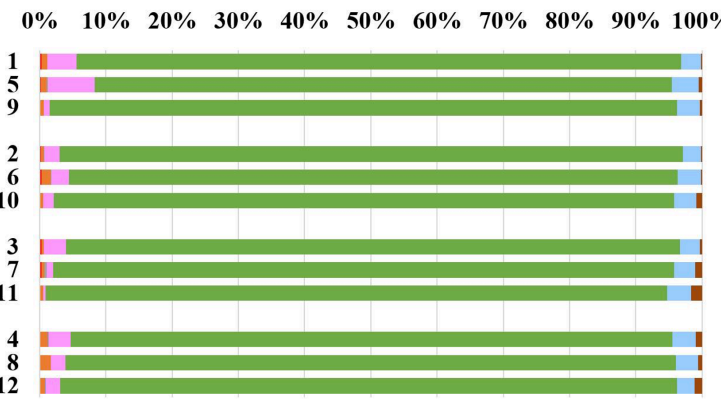

Figure 7 Male subjects' 8 emotions comparison

$\begin{array}{lllllllllll}0 \% & 10 \% & 20 \% & 30 \% & 40 \% & 50 \% & 60 \% & 70 \% & 80 \% & 90 \% & 100 \%\end{array}$

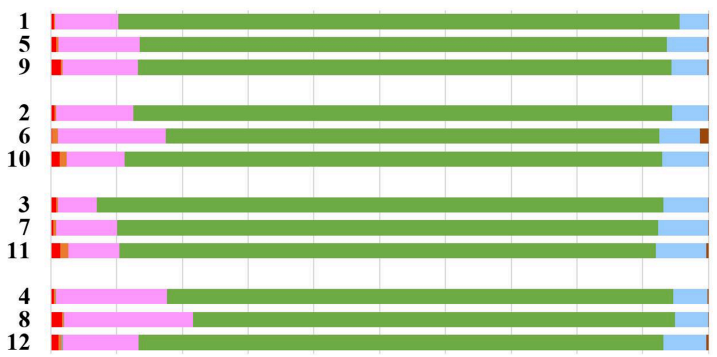

Figure 8 Female subjects' 8 emotions comparison

expression in the SUGO-MIMI condition) with $4.05 \%$, followed by 11 (sad expression in the static ears condition), with $3.60 \%$. The percentage of sad expression was larger in the SUGO-MIMI and static ears conditions than in the no ears condition, suggesting that the sad expression was more easily conveyed when some ears were attached to the head.

The second highest ratio was in happiness. The largest change was in 5(normal expression in the SUGO-MIMI condition) with $7.08 \%$, followed by 1 (normal expression in the no ears condition) with $4.40 \%$. The SUGO-MIMI condition had the highest percentage of normal and angry expressions, but the no ears condition had the highest percentage of sad and happy expressions.

The third most significant change was in contempt. The highest ratio was 8 (happy expression in the SUGO-MIMI condition) with $1.64 \%$, followed by 6 (angry expression in the SUGO-MIMI condition) with $1.43 \%$. These results may be related to the opinions of some male subjects that moving ears felt like eyesores.

\subsection{Female subjects}

Figure 8 shows the emotion comparison results in female 
subjects. Based on happiness, which had the highest ratio other than neutral, the highest percentage was 8 (happy expression in the SUGO-MIMI condition) with 19.6\%, followed by 4 (happy expression in the no ears condition) with $16.8 \%$. For all expressions, the percentage of happiness was higher in the SUGO-MIMI condition than in the static ears or no ears conditions, suggesting that the presence of the SUGO-MIMI amplified and conveyed the joy.

The second highest percentage was in sadness. The largest change was observed in 11 (sad expression in the static ears condition) with $7.73 \%$, followed by 7 (sad expression in the SUGO-MIMI condition) with $7.61 \%$. In the sad expression, the sadness was transmitted more in the condition in which some ears were attached to the head than in the no ears condition

The third most significant change was in anger. The highest ratio was 8 (happy expression in the SUGO-MIMI condition) with $1.69 \%$, followed by 9 (normal expression in static ears condition) with $1.51 \%$. The stress of viewing 12 videos may have influenced the results since the percentage increased in the latter half of the experiment.

\section{CONSIDERATION}

Comparing the emotions of males and females, it was discovered that males perceived the overall facial expression more negatively, and females perceived it more positively. The proportion of emotions other than neutral, happiness, and sadness was not high for both males and females, but the proportion of contempt was slightly higher in men. In the presentation of sad expressions, both males and females were more likely to express sadness in the condition in which some ears were attached to the head. For the presentation of happy expressions, the joy was amplified in females, while the mimicry of joy was lower in males.

In addition, the percentage of neutral was lower in females overall. This result is consistent with the previous study [11], which showed that women are more likely to express their emotions than men.

\section{LIMITATION}

Due to the COVID-19, we could conduct the experiment only online and there is a possibility that the results would be different in a face-to-face experiment. However, the online experiment has some good aspects such as gathering subjects from various regions. Since the performer in videos used in the experiment was only a woman, gender differences may have occurred due to the influence of the gender of the performer. In the future, we would like to compare the effects of male and female performers. We would examine the case where the performer is a genderless neutral agent such as computer graphics characters and robots.

\section{CONCLUTION}

In this paper, we examined the effects of SUGO-MIMI, a device that aims to augment facial expressions by moving an ear-shaped plate in conjunction with the movement of eyebrows. As a result, the device was able to amplify and convey emotions in both men and women for sad expressions, and in women for happy expressions.

In the future, we would introduce emotional judgments not only for facial expressions, but also for questionnaire responses and speech content, and conduct comprehensive analysis.

\section{ACKNOWLEDGMENTS}

We would like to express our sincere gratitude to Dr. Kaisei Sakurai from DWANGO Co.,Ltd., for his guidance in composing the paper, and to Mr. Itaru Kawakami and Mr. Hirokazu Enya of Sugoi Lab for providing equipment.

We would also like to thank the 67 subjects, Kenichi Ito of The University of Tokyo, Rihito Tsuboi of Meiji University, Liu Yingqing and Yurie Suzuki, and Hikaru Sawatari of Kanazawa University. We would like to express our sincere gratitude to Prof. Kazushi Nishimoto of Japan Advanced Institute of Science and Technology (JAIST), Prof. Emeritus Susumu Kunifuji of JAIST, and all the others for their cooperation.

\section{REFFERENCES}

[1] S. Takagi; The role of facial expressions and body movements in communication, Bulletin of the Graduate School of Letters, Waseda University, vol.1, 51, pp. 25-36, 2005.

[2] M. Hidaka and K. Kosugi; A Social Psychological Study of the Expression "Reading the Air." Research Papers. Part 3, Arts, Physical Education, Education, Psychology, 62, pp.139-144, 2012.

[3] L. R. Finka, et al; Geometric morphometrics for the study of facial expressions in non-human animals, using the domestic cat as an exemplar, Scientific Reports, 9(1), pp.9883, 2019.

[4] J. Kaminski et al.; Evolution of facial muscle anatomy in dogs, Proceedings of the National Academy of Sciences, 116(29), pp.14677-14681, 2019.

[5] M. Masui et al. PerformEyebrow: A proposal of an artificial eyebrow shape control device that can extend facial expressions, Proceedings of the Information Processing Society of Japan(IPSJ), 2020-HCI-189(10), pp.1-7, 2020.

[6] newrowear. "necomimi," http://neurowear.com/projects_detail/necomimi.html_ (Check 2020-12-21)

[7] Y. Mitsukura; KANSEI Detection and Its Application Using the EEG: Widespread of KANSEI Research in Society, IEICE Fundamentals Review, 13(3), pp.180-186, 2020.

[8] P. Ekman; Facial expressions of emotion: New findings, new questions, Psychological Science, 3(1), pp.34-38, 1992.

[9] Y. Kakui et al.; A Method of Automatic Generation of YouTube Thumbnails and Its Evaluations, ICESE, 119(457), pp.157-162, 2020.

[10] S. Kimura, S. Kunifuji and T. Ito; A Comparative Study of the Effects of Clapping Hands Sounds and Gesture Presentation During Online Meetings, KICSS, Nov,2020.

[11] A. M. Kring and A. H. Gordon; Sex differences in emotion: Expression, experience, and physiology, Journal of Personality and Social Psychology, 74(3), pp.686-703, 1998. 